In der Rubrik „Literatur kompakt" werden die wichtigsten Originalarbeiten aus der internationalen Fachliteratur referiert.

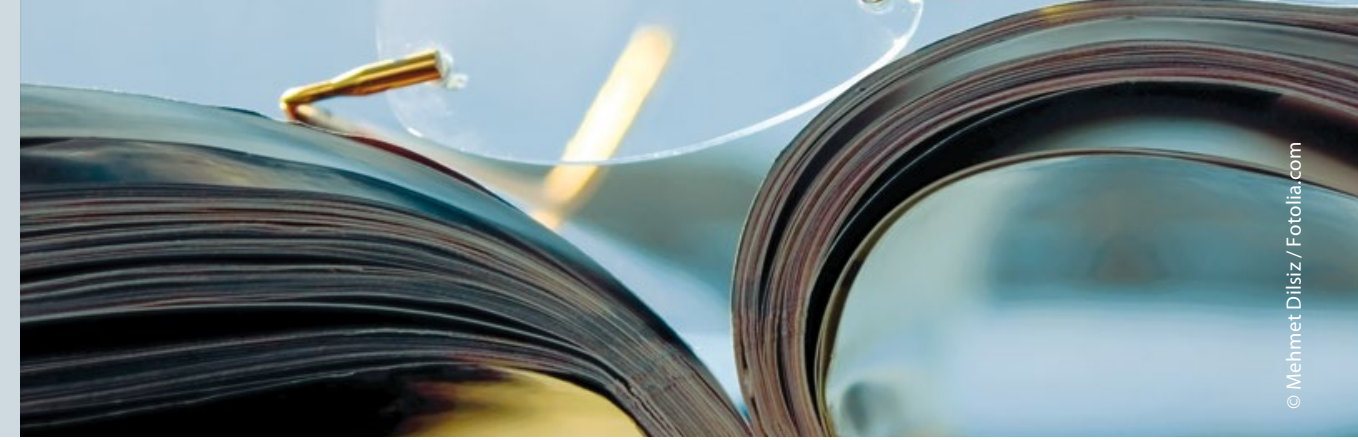

\section{Gefahr für Palliativpatienten durch MRSA?}

\section{Wie verbreitet MRSA auf Palliativstationen ist und welche Konsequenzen das hat, haben Forscher aus Wuppertal in einer prospektiven Studie untersucht.}

nfektionen mit multiresistenten Keimen stellen bei Palliativpatienten eine besondere Herausforderung dar. Diese Patienten zu isolieren ist nicht unproblematisch; denn dadurch würde sich die Betreuungsqualität deutlich verschlechtern. Bisher liegen allerdings nur wenige und zudem retrospektiv erhobene Daten zur Prävalenz von Methicillin-resistenten Staphylococcus-aureus-Stämmen (MRSA) in palliativmedizinischen Einrichtungen vor. Aufgrund dessen lässt sich nicht sagen, inwieweit eine Isolierung bei diesen Patienten überhaupt von Nutzen ist.

Das Team um Oliver Schmalz vom HELIOS Klinikum Wuppertal screente alle Patienten, die innerhalb von $13 \mathrm{Mo}$ naten in der onkologischen Palliativstation des Klinikums betreut wurden, auf MRSA und verglich die Ergebnisse mit den Patienten der anderen Stationen.

281 Palliativpatienten waren an der Studie beteiligt, von 269 Patienten lagen verwertbare Datensätze vor. Bei ihnen hatte man innerhalb von 48 Stunden nach Aufnahme in der Palliativstation Abstriche von Nase, Rachen und Leiste sowie gegebenenfalls von Wunden gemacht. Sämtliche Abstriche wurden bei den Studienteilnehmern wöchentlich wiederholt. Viermal täglich wurden die Patienten nach Symptomen wie Schmerzen, Übelkeit, Atemnot, Müdigkeit und Schwäche befragt.

Ein einmaliges MRSA-Screening bei Aufnahme erhielten im selben Zeitraum auch alle anderen Patienten des Klinikums; insgesamt waren es auf allen nicht palliativen Stationen zusammengenommen 11.150. Beim Transfer von Patienten aus einer anderen Station auf die Palliativstation wurden diese zusätzlich noch einmal mit der beschriebenen Prozedur gescreent.

Die im Mittel 69,7 Jahre alten Palliativpatienten hatten einen durchschnittlichen Karnofsky-Index von $40 \%$, der Palliative-Prognostic-Score (PaP-S) lag im Mittel bei 5. Die Patienten befanden sich im Schnitt 9,7 Tage auf der Station und wurden in dieser Zeit zweimal einem MRSA-Screening unterzogen. Dabei war das Screening-Ergebnis in 8,5\%

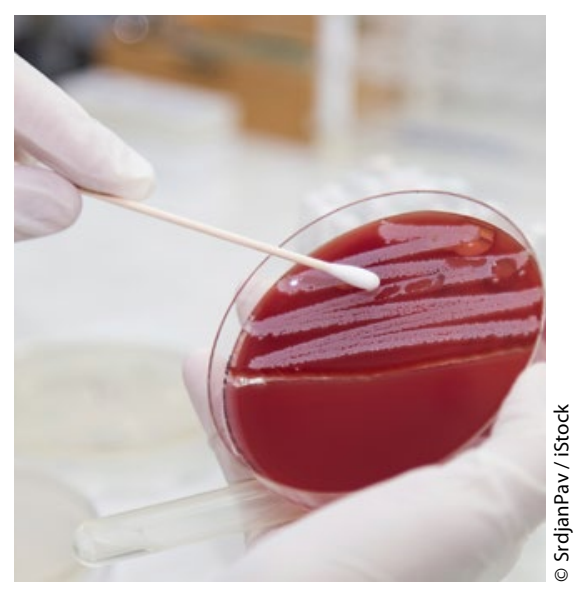

Die Prävalenz für MRSA ist auf Palliativstationen doppelt so hoch wie auf normalen Stationen. der Fälle (24 von 281) positiv. Die MRSAPrävalenz war damit auf der Palliativstation in etwa doppelt so hoch wie bei den Patienten der anderen Stationen (einschließlich der Intensivstation); auf diesen lag sie zusammengefasst bei 4,6 pro 100 Patienten.

Auf der Palliativstation des Klinikums Wuppertal kommt beim Nachweis einer MRSA-Besiedelung kein rigoroses Isolationsprotokoll zum Einsatz. Isoliert werden lediglich die Patienten mit MRSANachweis, bei denen Wundsekret austritt, die ein Tracheostoma haben oder bei denen Rachen oder Atemwege besiedelt sind.

Dennoch, so betonen Schmalz und Kollegen, hatte sich kein einziger Fall einer Übertragung von MRSA im Klinikum selbst ereignet. So blieben alle Patienten, die beim ersten Mal negativ gescreent worden waren, auch in allen weiteren Abstrichen negativ.

Fazit: Die Studie zeigt zum einen, dass auf Palliativstationen mit einer höheren MRSA-Prävalenz zu rechnen ist als auf anderen Stationen eines Allgemeinkrankenhauses. Allerdings legt die Erfahrung aus dem Klinikum Wuppertal nahe, dass das Übertragungsrisiko bei Palliativpatienten offenbar gering ist. Ursache hierfür, so wird spekuliert, ist möglicherweise die intensive Betreuung durch das Pflegepersonal sowie die Tatsache, dass die meisten Patienten nicht in der Lage sind, ihr Bett zu verlassen.

Elke Oberhofer

Schmalz $\mathrm{O}$ et al. Methicillin-resistant Staphylococcus aureus in palliative care. Palliative Medicine, online 29. April 2016 